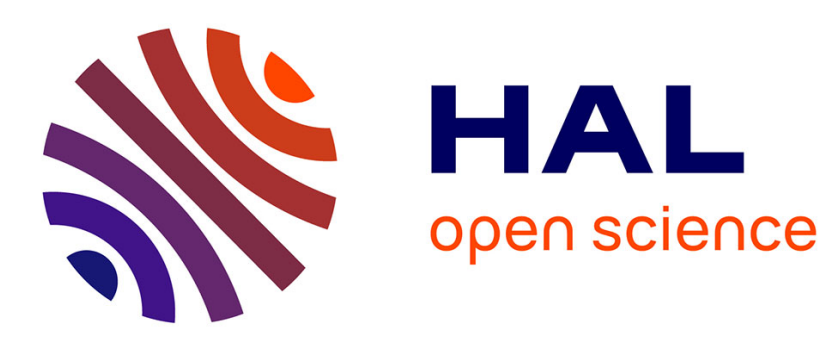

\title{
Etude du bruit acoustique d'arcs électriques; cas des fours à arcs industriels
}

\author{
H. Beji, M. Fitaire, J. Antoine
}

\section{To cite this version:}

H. Beji, M. Fitaire, J. Antoine. Etude du bruit acoustique d'arcs électriques; cas des fours à arcs industriels. Revue de Physique Appliquée, 1983, 18 (12), pp.795-802. 10.1051/rphysap:019830018012079500 . jpa-00245149

HAL Id: jpa-00245149

https://hal.science/jpa-00245149

Submitted on 1 Jan 1983

HAL is a multi-disciplinary open access archive for the deposit and dissemination of scientific research documents, whether they are published or not. The documents may come from teaching and research institutions in France or abroad, or from public or private research centers.
L'archive ouverte pluridisciplinaire HAL, est destinée au dépôt et à la diffusion de documents scientifiques de niveau recherche, publiés ou non, émanant des établissements d'enseignement et de recherche français ou étrangers, des laboratoires publics ou privés. 


\title{
Etude du bruit acoustique d'arcs électriques ; cas des fours à arcs industriels
}

\author{
H. Beji (*), M. Fitaire \\ Laboratoire de Physique des Gaz et des Plasmas (**), Bât. 212, Université Paris XI, 91405 Orsay Cedex, France
} et J. Antoine

Laboratoire d'Etude et de Contrôle de l'Environnement Sidérurgique (LECES), 57210 Maizières les Metz, France

(Reçu le 13 juin 1983, révisé le 16 septembre, accepté le 19 septembre 1983)

\begin{abstract}
Résumé. - Il est montré que la génération d'ondes acoustiques par des fours à arcs est due aux variations de la puissance électrique d'alimentation de ces fours; les valeurs instantanées de la fluctuation de pression générée sont, comme prévu par l'étude préalable en laboratoire, liées à la dérivée temporelle de cette puissance. On procède dans un deuxième temps à l'étude des effets d'ensemencement d'un arc par un alcalin, mettant ainsi en évidence la possibilité de réduire le taux de fluctuations de la puissance électrique cédée à l'arc et donc aussi le niveau sonore généré. L'étude met en outre en évidence une diminution de l'impédance de l'arc qui implique, à tension d'alimentation donnée, un accroissement de la puissance moyenne dans cet arc. Les conséquences industrielles de ce travail sont explicitées.
\end{abstract}

\begin{abstract}
We show that the generation of acoustic waves by arc furnaces is due to the variations of the electric power supplied. The instantaneous values of the pressure fluctuations are, as predicted by previous laboratory studies, related to the time derivative of this power. We then study the effects of the seeding of an arc with an alkaline and show that the fluctuations rate of the power can be reduced and, therefore, the generated acoustic noise. Our study also shows that the electric impedance of the arc is reduced, implying that, at constant voltage, the mean electric power to the arc is increased. Descriptions of possible industrial applications are given.
\end{abstract}

\section{Introduction.}

Il est connu [1, 2] que l'amplitude d'ondes acoustiques excitées dans un plasma est, toutes choses égales par ailleurs, proportionnelle à la dérivée temporelle de l'énergie cédée au gaz neutre par les particules chargées du plasma.

Les émissions acoustiques des fours à arcs utilisés par l'industrie sidérurgique sont donc une conséquence du caractère instable de ces arcs, instabilités qui ont en outre des répercussions importantes sur le fonctionnement même des fours.

Dans ce qui suit, est tout d'abord établie une corrélation entre, d'une part, les valeurs instantanées des signaux sonores émis par des fourcs à arcs et, d'autre

$\left(^{*}\right)$ Adresse actuelle : Institut Technologique d'Architecture et d'Urbanisme d'Art, Route de l'Armée Nationale, Tunis, Tunisie.

$\left({ }^{* *}\right)$ Laboratoire Associé au Centre National de la Recherche Scientifique (C.N.R.S.). part, la puissance électrique totale qui leur est cédée ; ceci est réalisé à l'aide du four à arcs pilote de l'IRSID alimenté en courant continu et d'un four industriel en fonctionnement normal.

Cette corrélation mise en évidence, il est recherché un procédé de réduction de ces émissions acoustiques, c'est-à-dire une réduction du taux d'instabilité électrique des fours : on crée artificiellement une atmosphère plus aisément ionisable que l'air à la pression atmosphérique en ensemençant l'arc avec des sels alcalins (procédure déjà utilisée [3], mais alors dans un but opposé, bien que l'idée physique simple sur laquelle elle reposait fût la même). Mise en œuvre en laboratoire par différentes voies d'approche, cette méthode a été essayée sur un four et les résultats obtenus dans ces conditions se sont révélés prometteurs.

On verra que la réduction du taux d'instabilités des fours à arcs peut avoir des conséquences, certes secondaires par rapport au problème ici étudié, mais essentielles sur le plan de la productivité et des nuisances de ces fours. 


\section{Rappels concernant l'excitation des ondes acoustiques dans les plasmas.}

La théorie de l'excitation d'ondes acoustiques repose sur l'utilisation des équations de l'hydrodynamique des gaz. Partant des équations de conservation de la densité et de la quantité de mouvement des neutres, équations associées à deux équations d'état du gaz on obtient, en négligeant la viscosité de celui-ci :

$$
\nabla^{2} p=\frac{1}{c^{2}} \frac{\partial^{2} p}{\partial t^{2}}-\frac{\gamma-1}{c^{2}} \frac{\partial H}{\partial t}+\operatorname{div} \mathbf{F}-\frac{\partial Q}{\partial t}
$$

dans laquelle $H, \mathrm{~F}$ et $Q$ sont respectivement : 1) l'énergie cédée au gaz par unité de volume et de temps; 2) la force exercée sur le même volume unité ; 3) la masse de gaz ajoutée ou retirée par unité de volume et de temps.

Toute perturbation intervenant dans les transferts entre arc et gaz neutre tend donc à modifier l'équilibre local de pression. On peut montrer [5] que dans le cas des plasmas courants et en particulier des arcs, le terme de source des ondes acoustiques lié à $H$ est beaucoup plus grand que les deux autres $: a$ ) les forces dues aux électrons tendent à compenser celles dues aux ions et $\mathbf{F}$ intervient dans l'équation à travers sa divergence $; b$ ) le calcul numérique conduit à négliger l'effet de $Q$ devant celui de $H$.

Dans ces conditions l'équation de propagation des ondes acoustiques dans un plasma contient un terme de source proportionnel à $\partial H / \partial t$, terme généralement dépendant du temps et des coordonnées d'espace. Connaître à chaque instant la fonction $H(\mathbf{r})$ est en dehors de nos possibilités expérimentales et n'est d'ailleurs pas nécessaire si les sources peuvent être considérées comme ponctuelles, c'est-à-dire si les longueurs d'ondes prises en compte sont grandes devant les dimensions des arcs. Ceci correspond dans notre cas à des fréquences de l'ordre du kilohertz, fréquences limites effectivement étudiées dans la présente étude.

On écrira donc que $H(t)=\langle H(r, t)\rangle_{\mathrm{r}}$ et, en première approximation, que $H=\alpha W$ où $W$ est l'énergie électrique totale cédée à l'arc et $\alpha$ une constante de proportionnalité. On notera par ailleurs que, $c^{2}$ variant comme la température $T$ du gaz neutre dans l'arc, l'équation de propagation peut encore s'écrire :

$$
\frac{\partial^{2} p}{\partial t^{2}}-\nabla^{2} p=\beta \frac{1}{T} \frac{\partial W}{\partial t}
$$

où $\beta$ est, pour un arc donné, une constante de proportionnalité. A température $T$ constante, la mesure de $W(t)$ est donc nécessaire et suffisante pour connaître, à un facteur constant près, la fluctuation de pression $p$ à un instant $t$ quelconque et en chaque point de l'espace (le calcul incluant bien entendu la présence d'éventuelles parois).

\section{Etude de fours à arcs industriels.}

En imposant des perturbations de courant à un arc au carbone brûlant dans l'air, nous avons montré en laboratoire $[5,6]$ que les ondes acoustiques ainsi excitées satisfont l'équation 2 précédente.

Les résultats de ces études ont été transposés au cas des fours à arc industriels. On cherche donc à étudier les émissions acoustiques de fours industriels, et plus précisément à établir une corrélation entre l'amplitude des signaux acoustiques émis et la dérivée de la puissance électrique totale cédée aux fours.

Les mesures des paramètres électriques et acoustiques ont été effectuées et enregistrées sur deux fours : a) un four (Sté Cockerill, Hautmont) d'une capacité de $45 \mathrm{t}$ (cuve de 4,80 $\mathrm{m}$ de diamètre), $30 \mathrm{MVA}$, alimenté en triphasé et fonctionnant donc avec trois électrodes; b) le four pilote (6 t) de l'IRSID à Maizières-les-Metz, alimenté en courant continu et fonctionnant avec une seule électrode.

3.1 CAS D'UN FOUR ALIMENTÉ EN TRIPHASÉ. - La mesure des tensions au secondaire du transformateur d'alimentation est simple, mais les valeurs ainsi obtenues ne sont pas celles réellement appliquées aux bornes des arcs : elles négligent les chutes de potentiel dans les électrodes. En admettant néanmoins, ce qui est tout à fait justifié, que ces électrodes ont une longueur constante pendant la durée de la mesure, on convient ainsi que les valeurs mesurées sont, à des facteurs constants près, celles réellement appliquées; ces trois facteurs constants sont égaux si on suppose en outre, ce que nous ferons, que les trois électrodes ont la même longueur.

Les valeurs de courants et de tensions instantanés ainsi que les signaux acoustiques émis ont été mémorisés à l'aide d'un enregistreur magnétique à bande.

Nous avons ensuite procédé au laboratoire à la numérisation de ces grandeurs à l'aide d'un convertisseur analogique-digital à quatre voies d'entrées simultanées. Le convertisseur et sa mémoire sont connectés à un micro-ordinateur, à travers un interface CAMAC-GPIB (IEEE 583-IEEE 488). Les valeurs numériques ainsi transférées sont ensuite traitées pour obtenir $P(t)=\sum_{i=1}^{3} V_{i} I_{i}, i$ désignant les phases successives du réseau. Le calcul montre (Fig. 1) qu'il n'y a pas de similitude apparente entre la puissance électrique instantanée $P(t)$ et le signal acoustique. Compte tenu de ce qui précède, une telle ressemblance ne pourrait d'ailleurs être que fortuite : c'est la dérivée de $P(t)$ qui doit être comparée au signal acoustique. Néanmoins, compte tenu de la complexité de ces signaux, une telle comparaison serait difficile. C'est pourquoi la figure 2 représente directement la puissance électrique et l'intégrale du signal acoustique enregistré, figure sur laquelle les $a_{1}, a_{2}, \ldots, a_{5}, b_{1}, \ldots, c_{5}$ associent des valeurs instantanées de la puissance électrique $P(t)$ à divers instants à celles du signal acoustique intégré aux mêmes instants (ce signal étant avancé d'un temps correspon- 


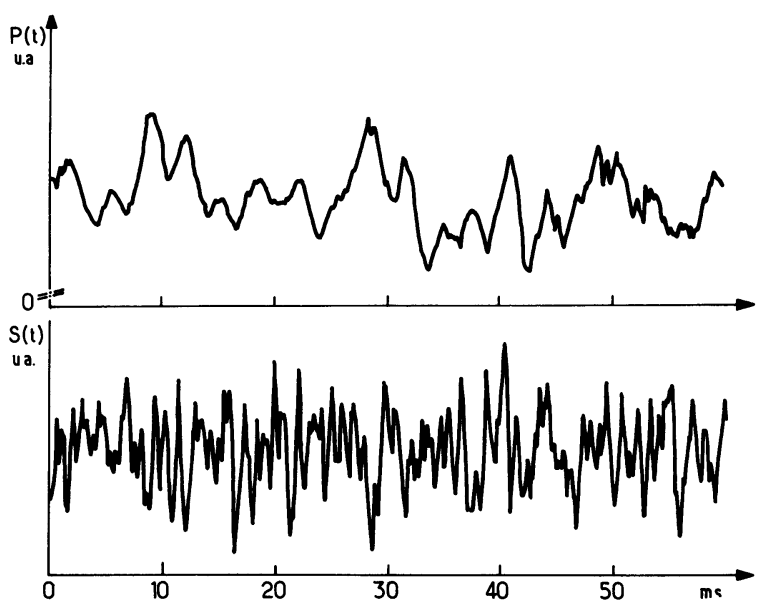

Fig. 1. - Variations de la puissance électrique totale $P(t)$ cédée au four et signal acoustique $S(t)$ reçu; l'origine de l'échelle des temps est fixée par le choix du début de digitalisation de $P(t)$. Le signal $S(t)$ est décalé par rapport à $P(t)$ d'un temps correspondant à celui du trajet moyen des ondes acoustiques entre le four et le microphone.

[Temporal variations of the electric power $P(t)$ supplied to the furnace, and received acoustic signal $S(t)$; temporal origin is at the beginning of $P(t)$ digitalization. Signal $S(t)$ is shifted with respect to $P(t)$ for a time interval equal to the mean propagation time between furnace and microphone.]
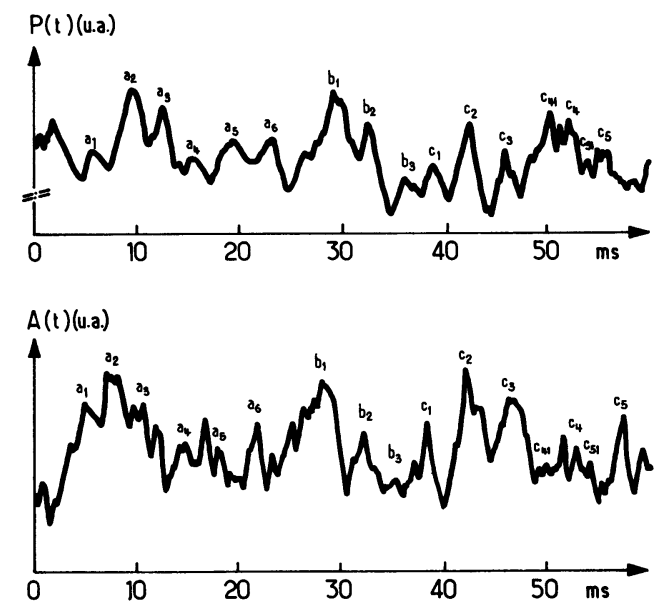

Fig. 2. - Variations de la puissance électrique totale $P(t)$ cédée $\mathrm{au}$ four et intégrale du signal acoustique $A(t)=\int_{0}^{t} S(t) \mathrm{d} t$. Les $a_{1}, a_{2}, \ldots, c_{5}$ associent, à des instants particuliers, des valeurs de $P(t)$ et de $A(t)$.

[Variations of the electric power $P(t)$ supplied to the furnace and the integral $A(t)=\int_{0}^{t} S(t) \mathrm{d} t$ of the acoustic signal. Specific values of $P(t)$ and $A(t)$ at specific times are indicated by $a_{1}, a_{2}, \ldots, c_{5}$.] dant au temps moyen de propagation). On observe alors une bonne ressemblance entre les dépendances temporelles de ces grandeurs; il n'y a néanmoins pas identité, identité à laquelle plusieurs phénomènes s'opposent :

1) le signal acoustique $A(t)$ reçu est somme de ceux créés au niveau de chacune des trois électrodes; ces électrodes étant toutes trois à des distances différentes du microphone, les chemins parcourus par ces signaux, et donc leurs temps de propagation, sont tous différents (le microphone est à $5 \mathrm{~m}$ de la porte du four et les électrodes sont disposées sur un cercle centré au milieu de la cuve de fusion elle-même d'un diamètre de l'ordre de 2,5 m). Dans ces conditions $A(t)$ prend en compte, à un instant donné, des signaux émis en des temps différents (on notera d'ailleurs que le décalage temporel entre les $a_{1}, \ldots, c_{5}$ de $P(t)$ et l'intégrale de $A(t)$ n'est pas constant).

2) Dans la phase de fusion des ferrailles qui correspond à la figure 2 , les trois électrodes creusent chacune un puits dans ces ferrailles; ces puits n'ont pas, à un instant donné, la même profondeur : $a$ ) les impédances électriques de trois arcs sont différentes ainsi donc que la puissance électrique associée ; $b$ ) les signaux acoustiques émis dans ces puits ont à parcourir des trajets complexes et tous différents pour atteindre le microphone.

Le calcul de la fonction d'intercorrélation entre le bruit intégré et la puissance électrique cédée aux trois arcs, et dont le résultat est montré figure 3 , indique une

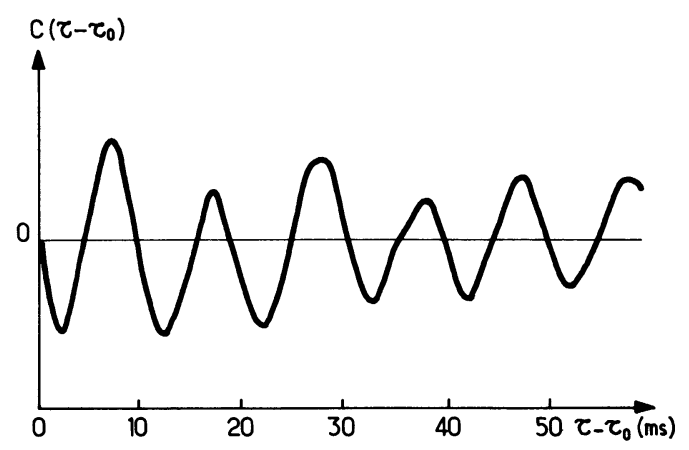

Fig. 3. - Fonction d'intercorrélation $C\left(\tau-\tau_{0}\right)=$ $\int_{-\infty}^{+\infty} A\left[t-\left(\tau-\tau_{0}\right)\right] P(t) \mathrm{d} t$ entre l'intégrale $A(t)$ du signal acoustique et la puissance électrique totale $P(t) ; \tau_{0}$ représente le temps de propagation de l'onde entre sa source et le microphone; $\tau_{0}$ est ajusté à la valeur moyenne de ce temps de propagation puis, arbitrairement, pour que $C(0)=0$.

[Intercorrelation function $C\left(\tau-\tau_{0}\right)=\int_{-\infty}^{+\infty} A\left[t-\left(\tau-\tau_{0}\right)\right] \times$ $P(t) \mathrm{d} t$ between acoustic signal integral $A(t)$ and total electric power; $\tau_{0}$ is the propagation time between source and microphone; $\tau_{0}$ is adjusted to the mean value of the propagation time, and arbitrarily for $C(0)=0$.] 
bonne cohérence et confirme que le bruit acoustique d'un four dépend de la dérivée de la puissance électrique qui lui est fournie.

\subsection{CAS D'UN FOUR À UNE SEULE ÉLECTRODE ALIMENTÉE} PAR UNE TENSION ALTERNATIVE REDRESSÉE. - Compte tenu de ce qui précède, on s'attend à ce qu'un four alimenté par une tension continue ne génère pas d'ondes acoustiques; ce n'est pas ce que l'on observe, la tension d'alimentation n'étant bien entendu pas totalement filtrée après redressement et l'arc lui-même n'étant pas parfaitement stable.

La figure 4 montre les variations de la puissance électrique cédée à l'arc et celles de l'intégrale du bruit acoustique reçu par un microphone disposé comme précédemment (ces variations sont encore obtenues par enregistrement magnétique et traitement numérique). La correspondance entre ces variations est bien meilleure que celle trouvée avec une alimentation triphasée : il existe une seule source d'ondes acoustiques, permettant ainsi d'établir plus simplement cette correspondance.
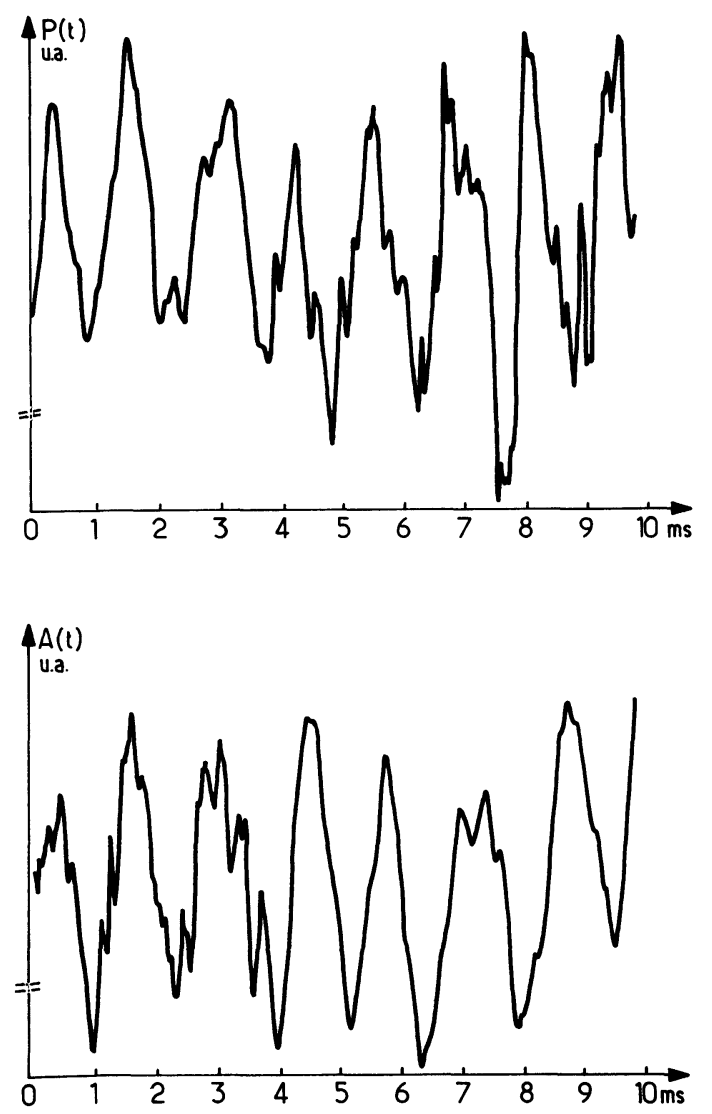

Fig. 4. - Variations de la puissance électrique cédée à l'arc et intégrale $A(t)$ du signal acoustique; l'alimentation est obtenue par redressement à partir du réseau triphasé et une seule électrode est utilisée.

[Variations of the electric power $P(t)$ supplied to the arc and integral $A(t)$ of the acoustic signal; the three phases are rectified and connected to one electrode.]
On observera un décalage variable entre la puissance électrique et le signal intégré, décalage représentatif des mouvements de la tache cathodique : en s'approchant du microphone la source réduit le temps de propagation et réciproquement lorsqu'elle s'en éloigne. Les déplacements ainsi mesurés sont de l'ordre de la dizaine de centimètres, conformes à ceux observables visuellement sur l'extrémité de l'électrode dont le diamètre est de $25 \mathrm{~cm}$.

On conclura, de ce qui précède, que les signaux acoustiques générés par les fours à arcs ont pour origine les variations de la puissance électrique d'alimentation de ces fours et, plus précisément, que l'amplitude des ondes excitées est liée à la dérivée temporelle de cette puissance électrique. Une telle conclusion était attendue compte tenu des résultats acquis en laboratoire [6]. Néanmoins, et à cause de la complexité du milieu étudié, il n'est pas ici possible d'établir une stricte proportionnalité entre les grandeurs concernées; de forts gradients de température existent dans la cuve du four et les taches cathodiques sont mobiles : ceci a pour effet de modifier à chaque instant la valeur de cette constante de proportionnalité.

\section{Ensemencement d'arcs par du potassium ; essais de réduction du bruit émis.}

Compte tenu de ce qui précède, il est clair que la réduction des niveaux sonores générés par des fours à arcs suppose une réduction de la dérivée de la puissance électrique d'alimentation et/ou une augmentation de la température du four.

On observe sur un four industriel en fonctionnement des fluctuations rapides de la puissance électrique, fluctuations dues aux instabilités propres de l'arc et tout particulièrement aux mouvements aléatoires des taches cathodiques. On observe en outre que chaque arc s'allume et s'éteint deux fois par alternance : il en résulte inévitablement dans le spectre de bruit acoustique des signaux à $100 \mathrm{~Hz}$ et aux harmoniques de cette fréquence.

En ce qui concerne ce dernier point, notons que la dérivée de la puissance électrique est d'autant plus grande que les allumages et extinctions se produisent pour des tensions plus élevées. Il y a donc lieu, au moins pour cette raison, de faciliter le fonctionnement des arcs; c'est ce qui a été essayé en ensemençant la colonne de ces arcs avec un alcalin (bas potentiel d'ionisation), et la suite de l'étude concerne les conséquences sur le fonctionnement des arcs d'un tel ensemencement avec du potassium.

Dans un premier temps on comparera les courants et tensions de fonctionnement d'un arc alimenté en alternatif, arc produit avec et sans potassium entre deux électrodes en graphite. L'expérience sera prolongée par l'utilisation d'un montage permettant de de superposer des impulsions de courant à un courant continu et de mettre en évidence les effets de cet ensemencement sur les principaux paramètres de la décharge. 
4. 1 EFFETS DE POTASSIUM SUR UN ARC FONCTIONNANT EN ALTERNATIF. - La figure $5 a$ montre la dérivée de la puissance électrique d'alimentation d'un arc fonctionnant entre deux électrodes en graphite $(\varnothing=8 \mathrm{~mm})$ et le signal acoustique $S(t)$ émis par cet arc (les électrodes sont disposées verticalement). On retrouve la corrélation, maintenant bien connue, entre ces deux quantités. Les mêmes enregistrements effectués lorsque l'on pulvérise dans l'arc une solution saturée de $\mathrm{KOH}\left(\simeq 1 \mathrm{~cm}^{3} / \mathrm{min}\right.$.) permettent d'obtenir la figure $5 b$.

En comparant ces deux figures, on observe que la présence du $\mathrm{KOH}$ a les effets suivants :

a) moindres fluctuations de la dérivée de puissance, indiquant ainsi un fonctionnement plus régulier de l'arc;

b) disparition, une demi-alternance sur deux, du pic associé à l'allumage de l'arc; ceci s'explique par le fait que la convection thermique tend à faire monter les fines gouttelettes de $\mathrm{KOH}$ vers l'électrode supérieure et qu'elles y sont plus efficaces lorsque cette électrode est cathode que lorsqu'elle est anode ;

c) la variation de puissance à l'allumage, lorsque celui-ci a lieu, est plus grande que précédemment ; ceci
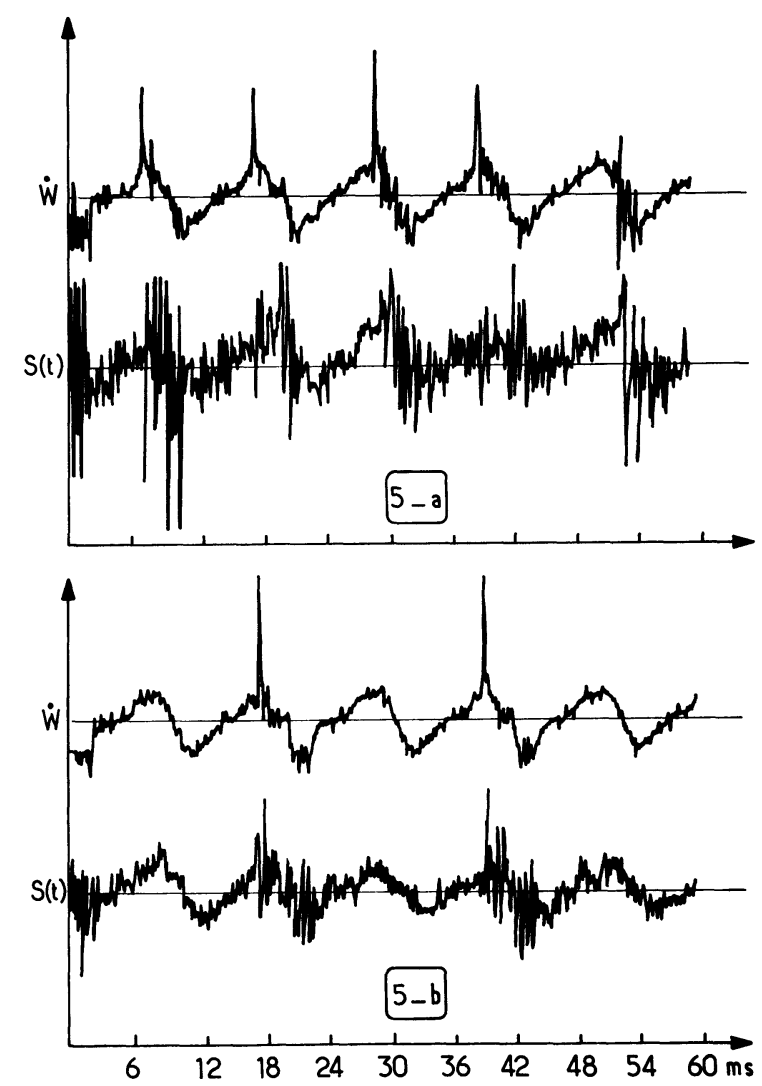

Fig. 5. - Dérivée de la puissance électrique et signal acoustique $S(t)$ avec, $(5 a)$, et sans $(5 b) \mathrm{KOH}$. Arc alimenté en alternatif $(50 \mathrm{~Hz}), 40 \mathrm{~V}, 40 \mathrm{~A}$ crête.

[Derivative of the electric power and acoustic signal $S(t)$ with $(5 a)$, and without $(5 b) \mathrm{KOH}$. The arc power source is at $50 \mathrm{~Hz}$.] montre que, même lorsque le $\mathrm{KOH}$ ne joue pas de rôle au niveau de la cathode, la puissance cédée à l'arc est plus grande;

d) le rapport des dérivées de puissance et des niveaux sonores qui leur sont associés montre une élévation de la température de l'arc de l'ordre de $20 \%$.

Compte tenu des points a et $\mathrm{d}$ précédents, on comprend que le niveau sonore mesuré soit moindre qu'en l'absence de $\mathrm{KOH}$. Pour éviter la dissymétrie de l'arc qui vient d'être observée, un autre procédé d'ensemencement a été mis en œuvre. Il consiste à percer un canal axial $(\varnothing=1 \mathrm{~mm})$ dans chacune des électrodes précédentes, canal dans lequel on fait couler du $\mathrm{KOH}$ anhydre chauffé jusqu'à fusion, $\mathrm{KOH}$ qui se solidifie ensuite après refroidissement.

Avec de telles électrodes, le niveau sonore de l'arc est à peine supérieur au bruit de la salle d'expérience $(\simeq 60 \mathrm{~dB})$. La comparaison des caractéristiques électriques de cet arc et de celles obtenues avec des électrodes homogènes est intéressante (Fig. 6). On observe plusieurs points importants résultant de la présence du potassium dans la décharge :

- le courant varie en suivant la tension sans irrégularité notable ;
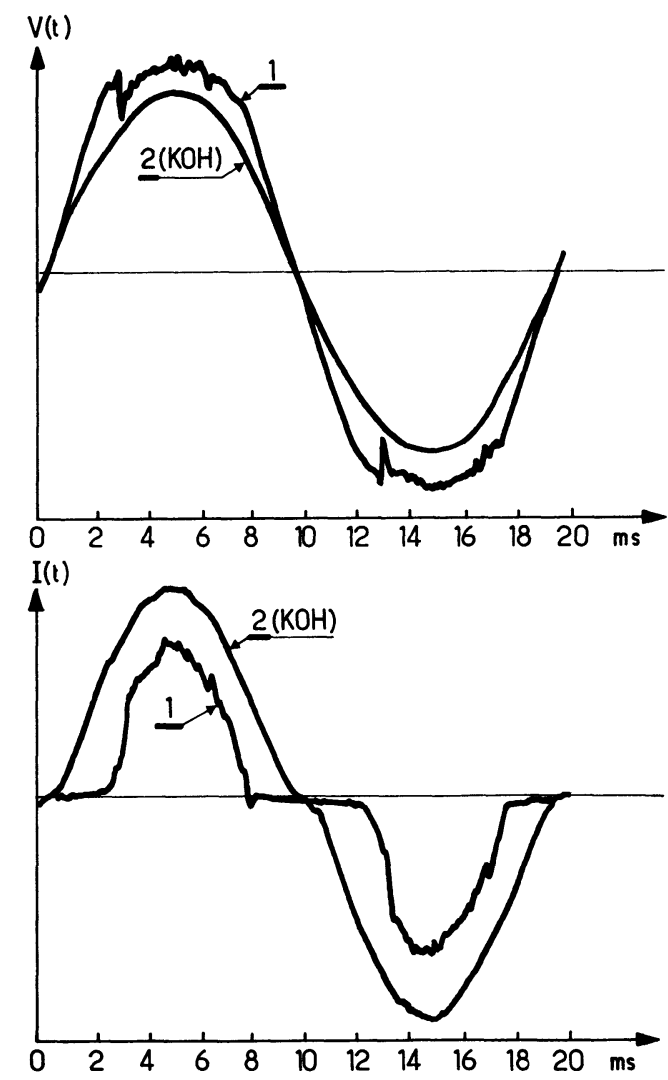

Fig. 6. - Comparaison des variations de la tension $V(t)$ et du courant $I(t)$ aux bornes d'un arc avec électrodes en graphite (1) et avec électrodes à canal de potassium (2).

[Comparison of voltage, $V(t)$, and current, $I(t)$, variations of an arc with graphite electrodes (1) and with electrodes having a potassium core (2).] 
- l'arc s'allume pour une tension très faible et, bien entendu, s'éteint pour une tension encore plus basse ;

- l'amplitude du courant est plus élevée, bien que celle de la tension soit, à cause de l'impédance interne de la source, plus faible : l'impédance électrique de l'arc a donc diminué :

- les deux électrodes jouent des rôles identiques.

En conclusion de ce paragraphe, on notera l'effet favorable du potassium sur le fonctionnement d'un arc alimenté en alternatif, effet observé pour l'une et l'autre méthode d'ensemencement.

4.2 EFFETS DU POTASSIUM SUR UN ARC PERTURBÉ PAR DES IMPULSIONS DE COURANT. - Un arc électrique alimenté par une source de courant est soumis à des impulsions de courant (Fig. 7). Un générateur (G) fournit ces impulsions qui sont amplifiées par un amplificateur $\left(\mathrm{A}_{1}\right)$ puis superposées au courant de la source continue $(S)$. Un multiplicateur analogique $\left(M_{1}\right)$ donne sur sa borne de sortie une tension proportionnelle à la valeur de la puissance instantanée fournie à l'arc, tension qui est digitalisée sur l'une des 4 voies d'entrée du convertisseur A/D précédemment utilisé. Ce même digitaliseur convertit des signaux représentatifs du courant et de la tension d'arc ainsi que le signal à la sortie d'un microphone $\left(\mathrm{M}_{2}\right)$ qui détecte les ondes acoustiques émises par cet arc.

Avec ce dispositif on retrouve la correspondance entre d'une part la dérivée de la puissance électrique fournie à l'arc, et d'autre part l'amplitude des ondes émises : la figure 9 montre une bonne similitude entre ces grandeurs.

L'amplitude de l'onde est, pour une même dérivée de puissance, plus faible à la descente de l'impulsion qu'à

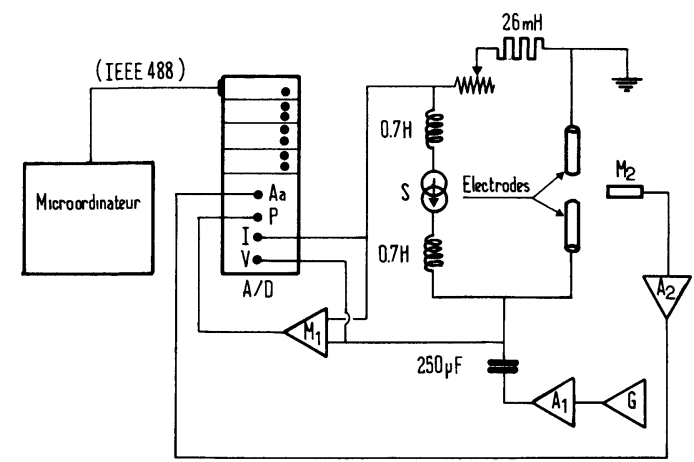

Fig. 7. - Dispositif d'étude complémentaire de l'effet du potassium sur un arc électrique. $A_{1}$ : amplificateur de puissance; $A_{2}$ : amplificateur de mesure; $M_{1}$ : multiplicateur analogique $(V \times I) ; \mathbf{M}_{2}:$ microphone à condensateur (Brüel et Kjaer, 1/8"); A/D : convertisseur analogique digital 4 voies de 10 bits, $32 \mathrm{kMots} ; \mathrm{S}$ : source de courant.

[Complementary experiment to study the effect of potassium on an electric arc. $\mathrm{A}_{1}$ : power amplifier; $\dot{A}_{2}$ : measurement amplifier; $\mathrm{M}_{1}$ : analog multiplier $(V \times I) ; \mathrm{M}_{2}$ : condenser microphone (Brüel and Kjaer, 1/8"); A/D : 10 bits analog to digital converter, 4 inputs, 32 kwords; $\mathrm{S}$ : current-regulated source.]

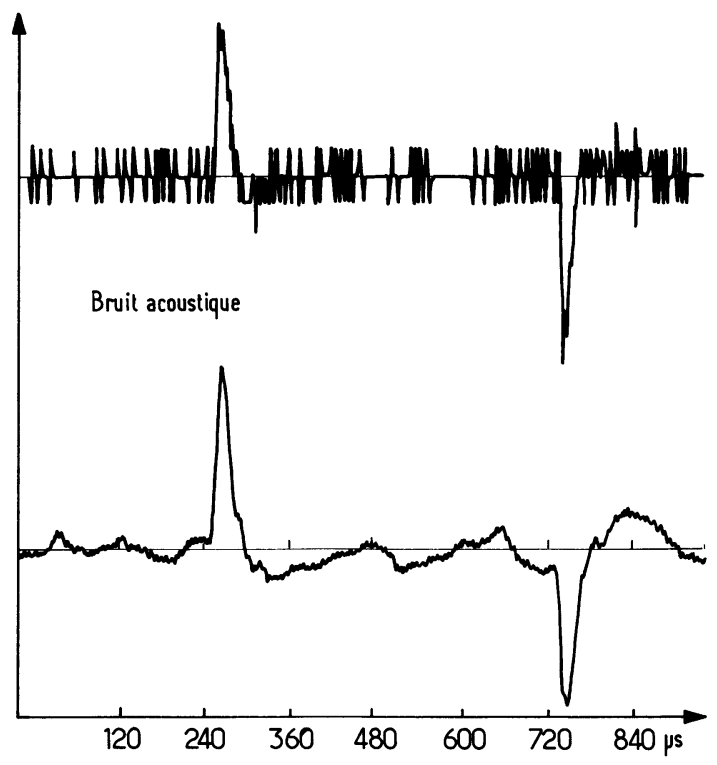

Fig. 8. - Dérivée de la puissance électrique fournie à l'arc et signal acoustique généré; $30 \mathrm{~V}, 40 \mathrm{~A}$ continus, impulsion de $20 \mathrm{~A}$.

[Time derivative of the electric power supplied to the arc and generated acoustic signal.]

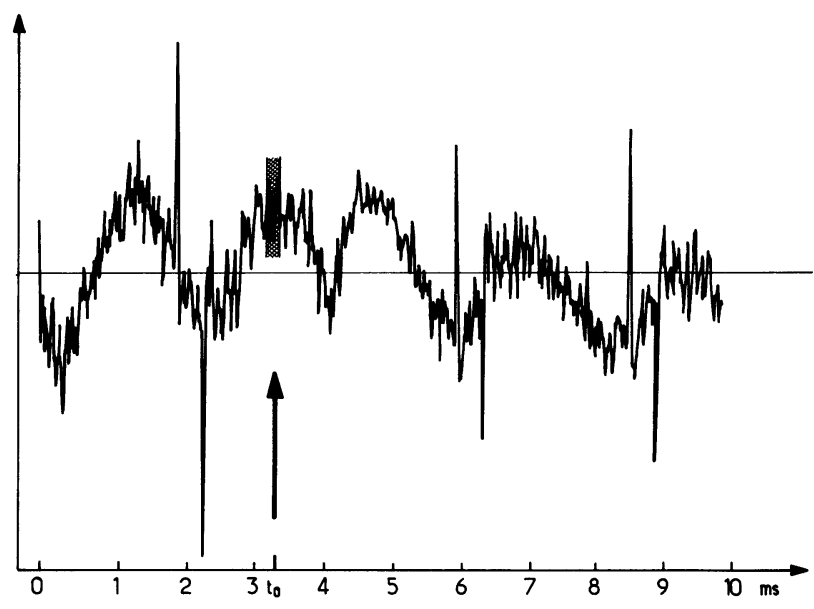

Fig. 9. - Signal acoustique enregistré pour des électrodes en graphite homogène $\left(t<t_{0}\right)$ et des électrodes à mèche; les valeurs des courants d'arc sont maintenues constantes.

[Acoustic signal for graphite electrodes $\left(t<t_{0}\right)$ and for electrodes with a potassium core $\left(t<t_{0}\right)$; the arc current is constant.]

la montée. En effet, ainsi que le prévoit la théorie, l'amplitude de l'onde est inversement proportionnelle à la température du gaz; à la montée de l'impulsion, la température de l'arc croît à partir d'une température $T_{0}$ et atteint, à la fin de cette impulsion, une température $T_{1}>T_{0}$ : l'amplitude du signal acoustique excité à cet instant est donc nécessairement plus faible qu'à la montée pour une même dérivée de la puissance électrique ; ceci permet de mesurer [6] $T_{1} / T_{0}$. 
Il est possible d'étendre l'étude de façon à comparer les caractéristiques de l'arc fonctionnant entre deux électrodes en graphite homogène $(\varnothing=8 \mathrm{~mm})$ à celles de l'arc dont les mêmes électrodes comportent une mèche de $\mathrm{KOH}$. Pour cela on enregistre, pendant un temps correspondant à l'utilisation d'une fraction (4 kMots) de la mémoire du convertisseur A/D, les données à digitaliser lorsque l'arc est produit entre deux électrodes homogènes. Dans un deuxième temps, et après avoir substitué à ces électrodes celles à mèche de $\mathrm{KOH}$, on fait circuler les mêmes courants que précédemment en reproduisant autant que faire se peut la distance interélectrodes obtenue à la fin de la première fraction de l'enregistrement. On constate (Fig. 9) une diminution nette de l'amplitude des ondes acoustiques générées lors de l'utilisation d'électrodes à mèche de $\mathrm{KOH}$, pour le même courant continu et la même impulsion de courant. On observe en outre une chute de la tension d'arc, tant statique que dynamique, de l'ordre de $45 \%$ (et bien entendu de la puissance cédée à l'arc). On peut vérifier que le rapport des dérivées des puissances instantanées est supérieur au rapport des amplitudes du bruit acoustique : la température de l'arc est plus élevée avec des électrodes à mèche qu'avec des électrodes en graphite homogène.

La figure 10 montre les variations de l'amplitude des ondes acoustiques excitées par des impulsions de courant en fonction de l'amplitude de ces impulsions et pour différentes puissances moyennes (l'amplitude des ondes acoustiques est mesurée à la montée des impulsions de courant). Chaque impulsion positive de

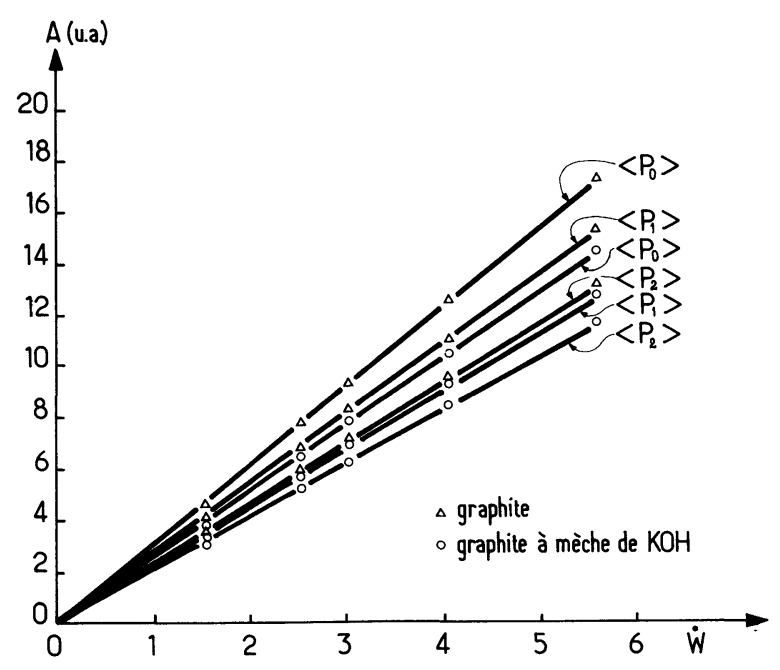

Fig. 10. - Amplitude des ondes acoustiques excitées par des impulsions de courant et à puissance électrique moyenne $P$ constante $\left(P_{0}=500 \mathrm{~W}, P_{1}=1 \mathrm{~kW}, P_{2}=3 \mathrm{~kW}\right)$, pour des électrodes en graphite et à mèche de $\mathrm{KOH}$.

[Amplitude of acoustic waves excited by current pulses at constant mean electric power $\left(P_{0}=500 \mathrm{~W}, P_{1}=1 \mathrm{~kW}\right.$, $\left.P_{2}=3 \mathrm{~kW}\right)$ for graphite electrodes and electrodes with a potassium core.] courant est suivie d'une impulsion négative de même amplitude et même durée de façon à maintenir une puissance moyenne constante, indépendante de la fréquence de répétition de ces impulsions et de leur amplitude. On retrouve sur cette figure une dépendance linéaire de l'amplitude des ondes avec la dérivée de puissance. On constate en outre que la pente des droites diminue lorsque la puissance moyenne cédée à l'arc augmente et que donc la température de celui-ci croît. La comparaison de ces pentes, à puissance moyenne constante, dans le cas d'électrodes en graphite et dans celui d'électrodes à mèche, montre que la température de l'arc augmente, en présence de $\mathrm{KOH}$, d'environ $45 \%$. La réduction, à courant constant, de la tension de fonctionnement qui résulte de l'utilisation de potassium est conforme à l'observation faite au paragraphe 4.1 .

4. 3 Application auX fours À ARCS. - La présence de potassium dans la décharge d'arc a pour première conséquence une élévation de la température d'arc et donc, toutes choses égales par ailleurs, une réduction des niveaux sonores émis. En outre, la stabilité de l'arc est améliorée, ce qui concourt à une réduction de ces niveaux sonores.

On notera néanmoins que la réduction des niveaux sonores de $120 \mathrm{~dB}$ à une valeur admissible de $90 \mathrm{~dB}$ supposerait une diminution du terme de source de ces ondes d'un facteur supérieur à 30 : une telle réduction n'a pu être obtenue. Par contre, l'abaissement de l'impédance d'arc permet un meilleur transfert d'énergie au four; en outre, l'allumage et l'extinction de l'arc alimentés en alternatif ayant lieu à tension plus basse, une meilleure utilisation de la source d'énergie électrique est obtenue : on accroît la puissance active cédée à la charge du four, ce qui devrait induire une réduction du temps de fusion des ferrailles et donc une meilleure productivité des fours. Un dernier point est à noter : diminuer le taux d'instabilités est aussi réduire les perturbations induites par les fours sur le réseau d'alimentation en énergie électrique.

Des essais préliminaires ont été entrepris [8] pour transposer la méthode précédemment décrite au cas des fours industriels. Par apport, soit de carbonate de potassium introduit directement dans la charge du four, soit d'un mélange de cérium et de lanthane introduit par l'intermédiaire d'une ou plusieurs mèches formées dans les électrodes, il a été observé : $a$ ) une réduction de 2 à $8 \mathrm{~dB}$ du niveau sonore dès qu'une phase liquide se constitue, aucune réduction notable n'étant mesurée pendant la phase de fusion des ferrailles; $b$ ) une diminution importante de la partie du spectre émis au-dessus de $500 \mathrm{~Hz} ; c$ ) dans certains cas une augmentation sensible de la puissance active cédée au four.

Les résultats de ces essais, sans démontrer l'intérêt de la méthode proposée, en sont néanmoins une indication positive. 


\section{Conclusion.}

Les valeurs instantanées des courants, des tensions et des émissions sonores d'un four industriel ont été enregistrées. Le traitement de ces grandeurs à l'aide d'un micro-ordinateur a montré une bonne similitude de forme entre les variations de la dérivée temporelle de la puissance électrique cédée au four et les émissions acoustiques de ce four. Cette bonne similitude, d'ailleurs attendue, a été vérifiée pour deux modes d'alimentation des fours.

Ce résultat acquis, nous avons entrepris l'étude des moyens de réduction des instabilités d'arc. Nous avons montré l'amélioration des conditions d'ionisation de la décharge d'arc résultant de l'utilisation de potassium.

Des résultats significatifs ont été obtenus en laboratoire, tant pour un arc alimenté en continu que pour un arc alimenté en alternatif. De manière générale l'arc présente une meilleure stabilité, et il en résulte donc des émissions sonores moins élevées. Les gains observés sur ce dernier point sont significatifs, sans être néanmoins très importants. Le but de cette étude était de trouver une voie d'action : ce but est atteint. Dès maintenant on peut affirmer que l'introduction d'un alcalin dans un plasma d'arc joue de manière favorable sur tous les paramètres électriques à prendre en compte : l'impédance de l'arc diminue, le temps compris entre l'instant d'extinction de celui de réallumage est diminué pour un fonctionnement en alternatif, enfin le courant d'arc est plus régulier en dehors de cet intervalle de temps. Il en résulte donc un meilleur transfert de l'énergie électrique à l'arc tout en obtenant un fonctionnement plus silencieux de celui-ci.

Bien entendu, la transposition de ces études au cas des fours industriels reste en partie à faire. Les premiers essais effectués par l'Institut de Recherches de la Sidérurgie (IRSID) confirment l'intérêt de la solution proposée. On notera d'ailleurs l'importance qu'aurait la mise au point d'un procédé industriel basé sur ces principes : il en résulterait, non seulement un fonctionnement beaucoup plus silencieux des fours, mais aussi, et peut-être surtout, une meilleure productivité de ces fours. Une stabilité accrue aurait en outre l'avantage de diminuer les perturbations induites sur le réseau de distribution d'énergie électrique et le "flicker" serait donc diminué, dans le même temps qu'il y aurait moins de puissance réactive à compenser. De tels avantages justifient que soient poursuivies les études en cours, études que le travail en laboratoire a orienté dans une direction prometteuse.

\section{Remerciements.}

Ce travail a été effectué dans le cadre de la R.C.P. 551 du C.N.R.S. et dans celui du Club "Arc Electrique " d'E.D.F.; l'un des auteurs (H.B.) a bénéficié d'une bourse de recherche du L.E.C.E.S. avec l'aide de la Direction des Affaires Sociales de la Communauté Européenne.

\section{Bibliographie}

[1] Fitaire, M. et Mantei, T., Phys. Lett. 29A (1969) 84.

[2] Fitaire, M. et Mantei, T., Phys. Fluids 15 (1972) 464.

[3] Fitaire, M. et Sinitean, E., Czech. J. Phys. B 22 (1972) 395.

[4] Yon Engel, A., Ionized Gases (Oxford Press) 1965.

[5] Dadgar, H., Thèse $3^{\mathrm{e}}$ cycle, Université Paris XI, Orsay (1977).
[6] Dadgar, H., Fitaire, M. et Pilorget, A., Rev. Gén. Elect. 88 (1979) 606.

[7] Antoine, J. et Fitaire, M., IXe Congrès de l'Union Internationale d'Electrothermie, Cannes (1980).

[8] Antoine, J., Engler, G. et Maurer, G., Rapport de fin d'étude à la C.E.C.A. (Réduction à la source du bruit du four à arc), IRSID L.E.C.E.S., Maizières-les-Metz (Nov. 1982). 